\title{
Papers
}

\section{Impact of DOTS compared with DOTS-plus on multidrug resistant tuberculosis and tuberculosis deaths: decision analysis}

Timothy R Sterling, Harold P Lehmann, Thomas R Frieden

\begin{abstract}
Objective This study sought to determine the impact of the World Health Organization's directly observed treatment strategy (DOTS) compared with that of DOTS-plus on tuberculosis deaths, mainly in the developing world.

Design Decision analysis with Monte Carlo simulation of a Markov decision tree.

Data sources People with smear positive pulmonary tuberculosis.

Data analysis Analyses modelled different levels of programme effectiveness of DOTS and DOTS-plus, and high $(10 \%)$ and intermediate $(3 \%)$ proportions of primary multidrug resistant tuberculosis, while accounting for exogenous reinfection.

Main outcome measure The cumulative number of tuberculosis deaths per 100000 population over 10 years.

Results The model predicted that under DOTS, 276 people would die from tuberculosis (24 multidrug resistant and 252 not multidrug resistant) over 10 years under optimal implementation in an area with 3\% primary multidrug resistant tuberculosis. Optimal implementation of DOTS-plus would result in four $(1.5 \%)$ fewer deaths. If implementation of DOTS-plus were to result in a decrease of just 5\% in the effectiveness of DOTS, $16 \%$ more people would die with tuberculosis than under DOTS alone. In an area with $10 \%$ primary multidrug resistant tuberculosis, $10 \%$ fewer deaths would occur under optimal DOTS-plus than under optimal DOTS, but 16\% more deaths would occur if implementation of DOTS-plus were to result in a $5 \%$ decrease in the effectiveness of DOTS

Conclusions Under optimal implementation, fewer tuberculosis deaths would occur under DOTS-plus than under DOTS. If, however, implementation of DOTS-plus were associated with even minimal decreases in the effectiveness of treatment, substantially more patients would die than under DOTS.
\end{abstract}

\section{Introduction}

The current recommendation for initial treatment of tuberculosis includes the standard first line regimen of isoniazid, rifampicin, pyrazinamide, and ethambutol. Since 1993 the World Health Organization has recommended that treatment be given as part of a comprehensive policy known as DOTS (directly observed treatment, short course; box). ${ }^{1}$ However, outcomes are poor when patients who are infected with Mycobacterium tuberculosis resistant to isoniazid and rifampicin (multidrug resistant tuberculosis) are treated with the standard regimen. ${ }^{2}$ Reserve or second line antituberculosis drugs have therefore become components of treatment regimens in national programmes. This approach is known as DOTS-plus (box). ${ }^{4}$ Although regimens based on reserve drugs have been reported to attain high rates of success of treatment in patients with multidrug resistant tuberculosis, ${ }^{56}$ the proposed widespread implementation of DOTS-plus has been controversial. ${ }^{7}$

In 1999, DOTS was used for $23 \%$ of smear positive cases of pulmonary tuberculosis globally. ${ }^{8}$ Second line agents that would be used under DOTS-plus are more expensive, more difficult to administer, and often poorly tolerated. Our hypothesis is that the implementation of DOTS-plus might divert resources from DOTS, decreasing the effectiveness of DOTS. In addition, if DOTS-plus were to be implemented incompletely the bacterium could develop resistance to second line agents, which would exacerbate rather than alleviate drug resistant tuberculosis.

A randomised controlled clinical trial assessing the effectiveness of DOTS compared with that of DOTS-plus is unlikely ever to be conducted because of logistical and ethical concerns. Since data on the effectiveness of both DOTS and DOTS-plus are available, however, we used decision analysis to compare the possible outcomes of the two treatment strategies and to assess the impact of varying levels of effectiveness.

\section{Methods}

We analysed data for HIV seronegative adults in the developing world who had smear positive pulmonary tuberculosis. Not enough data on the effectiveness of DOTS and DOTS-plus in HIV positive patients were available to be included in the analysis. We analysed DOTS and DOTS-plus for differing levels of effectiveness of the programmes, under conditions with moderate $(3 \%)$ and high (10\%) proportions of cases of
Johns Hopkins University Center for Tuberculosis Research, $424 \mathrm{~N}$ Bond Street, Baltimore, $\mathrm{MD}$ 21231, USA Timothy R Sterling associate professor of medicine and epidemiology

Department of Health Sciences Information, Johns Hopkins University School of Medicine, 2024 E Monument Street, Baltimore MD 21287-0007 Harold P Lehmann associate professor

New York City Department of Health, 125 Worth Street, New York, NY 10013, USA Thomas R Frieden commissioner of health

Correspondence to: T R Sterling tsterls@jhmi.edu

bmj.com 2003;326:574 
DOTS and DOTS-plus: treatment strategies for pulmonary tuberculosis in the developing world

DOTS is a package of five points:

- Commitment of governments to a national tuberculosis programme

- Detection of cases through case finding by sputum smear microscopy examination of patients with suspected tuberculosis in general health services

- Standardised short course chemotherapy with the first line drugs isoniazid, rifampicin, pyrazinamide, and ethambutol (or streptomycin) for, at least, all smear positive cases of tuberculosis under proper conditions of case management

- Regular, uninterrupted supply of all essential antituberculosis drugs

- A monitoring system for programme supervision and evaluation.

In addition:

- Mycobacterial cultures and drug susceptibility testing are not required

- Treatment is started on the basis of symptoms or a positive smear

- Second line drugs are not used

- Three categories of treatment regimens exist; all are directly observed

- In the developing world, mycobacterial cultures and susceptibility testing are generally not performed, so drug resistance is not detected even if it is present

\section{In DOTS-plus:}

- Second line antituberculosis drugs (more toxic and expensive, and less effective, than first line drugs) are used. The regimen includes two or more drugs to which the isolate is susceptible, including one drug given parenterally for six months or more. Total duration of treatment $18-24$ months; treatment is directly observed

- Treatment regimen is either:

Individualised according to drug susceptibility test results of the $M$ tuberculosis isolate identified on culture; or

Given as a standardised regimen to patients who fail supervised re-treatment (for example, when culture and drug susceptibility testing are not performed).

- Mycobacterial cultures and drug susceptibility testing may be performed.

incident multidrug resistant tuberculosis. We assessed the impact of the different treatment strategies by tabulating the cumulative number of tuberculosis deaths that occurred for each scenario. Because the impact of control programmes for tuberculosis on case rates and mortality usually becomes noticeable within 5-10 years of implementation, we assessed the effect of the two treatment strategies over a period of 10 years.

\section{Definitions}

We defined non-multidrug resistant tuberculosis as disease due to infection with $M$ tuberculosis with susceptibility to all antituberculosis agents or without resistance to isoniazid and rifampicin. We defined multidrug resistant tuberculosis as resistant to isoniazid and rifampicin, and highly drug resistant tuberculosis as resistant to isoniazid, rifampicin, and at least one second line antituberculosis agent (such as a fluo- roquinolone, ethionamide, para-aminosalicylic acid, cycloserine, or capreomycin).

\section{Model}

We used a Monte Carlo simulation of a Markov model (figure) to perform the decision analysis. ${ }^{9}$ For each scenario we followed a hypothetical cohort for 10 years, with a cycle length of one year. We defined the probability of each event for each cycle. For each analysis we performed 25000 Monte Carlo simulations and expressed the cumulative number of tuberculosis deaths as the rate per 100000 people during the 10 year period. To allow for a valid comparison between the different scenarios of treatment, and because the number of multidrug resistant and highly drug resistant outcomes was small, we used the same random sequence for all analyses. We used DATA version 3.5 (TreeAge Software, Williamstown, MA, USA) for our data analysis.

\section{Probability estimates}

We obtained probability estimates from articles in peer reviewed journals but excluded data published in abstract form only. We identified studies through a Medline search and obtained data from global reports published by WHO. Tables 1 and 2 show the estimates we used for the analyses of optimal DOTS and DOTSplus and the references for these estimates. When more than one value was present in different reports we used the mean value. Whenever possible we used data from studies conducted in the developing world. We excluded studies evaluating the effectiveness of DOTS-plus that included surgical management, to maintain comparability with studies evaluating the effectiveness of DOTS (which did not include surgical management). Because patients treated for prevalent multidrug resistant tuberculosis (previously treated patients who remain ill) have lower survival rates than patients treated for incident multidrug resistant tuberculosis ${ }^{10}$ we assumed that patients with prevalent multidrug resistant tuberculosis had a lower chance of cure without specific treatment than other untreated patients (for example, $10 \% v 20 \%$; table 1). Because of the greater extent of drug resistance we assumed that patients with highly drug resistant tuberculosis treated with DOTS-plus were less likely to be cured and to sur-

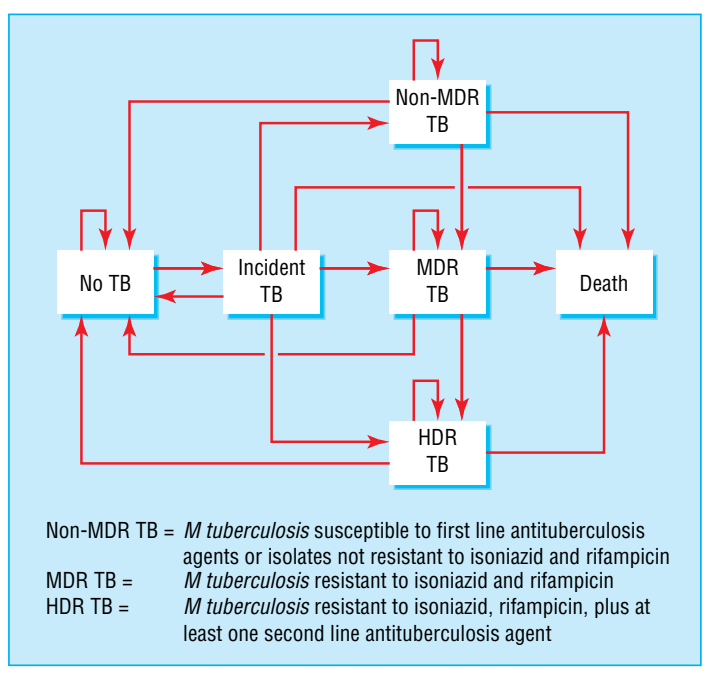

Transition states of the Markov analysis 
Table 1 Outcomes and probabilities of DOTS and DOTS-plus for smear positive pulmonary tuberculosis. Tuberculosis is incident unless otherwise indicated

\begin{tabular}{|c|c|c|c|}
\hline Type of tuberculosis & Outcome for patients & DOTS (\%) & DOTS-plus (\%) \\
\hline Multidrug resistant, non-multidrug resistant ${ }^{1}$ & Treated & 70 & 70 \\
\hline \multicolumn{4}{|l|}{ Treated: } \\
\hline Multidrug resistant & Alive & $91^{3}$ & $94^{11,12}$ \\
\hline Multidrug resistant & Cured & $47^{3}$ & $47^{11,12}$ \\
\hline Prevalent, multidrug resistant & Alive & $89^{3}$ & $94^{11,12}$ \\
\hline Prevalent, multidrug resistant & Cured & $21^{3}$ & $47^{11,12}$ \\
\hline Multidrug resistant & Highly drug resistant tuberculosis & 0 & $10^{13}$ \\
\hline \multicolumn{4}{|l|}{ Not treated: } \\
\hline Multidrug resistant, non-multidrug resistant ${ }^{14,15}$ & Alive & 50 & 50 \\
\hline Multidrug resistant, non-multidrug resistant ${ }^{16}$ & Cured & 20 & 20 \\
\hline Prevalent, multidrug resistant & Cured & 10 & 10 \\
\hline \multicolumn{4}{|l|}{ Treated: } \\
\hline Non-multidrug resistant ${ }^{17,18}$ & Alive & 96 & 96 \\
\hline Non-multidrug resistant $3,17,19$ & Cured & 79 & 79 \\
\hline Non-multidrug resistant ${ }^{2,20}$ & Multidrug resistant tuberculosis & $2 \dagger$ & $2 \dagger$ \\
\hline Prevalent, non-multidrug resistant ${ }^{17}$ & Alive & 94 & 94 \\
\hline Prevalent, non-multidrug resistant $3,17,19$ & Cured & 72 & 72 \\
\hline Highly drug resistant (treated) ${ }^{1}$ & Treated & - & 70 \\
\hline Highly drug resistant & Alive & - & 70 \\
\hline Highly drug resistant & Cured & - & 40 \\
\hline
\end{tabular}

${ }^{*} 12.5 \%$ when programme effectiveness decreases by $5 \%$, and $15 \%$ when programme effectiveness decreases by $10 \%$. Data not from developing world or pretreatment era.

†Assumes that the proportion of incident tuberculosis that is multidrug resistant is $3 \%$. This value is $4 \%$ when the proportion of incident tuberculosis that is multidrug resistant is $10 \%$.

vive than patients with multidrug resistant tuberculosis treated with DOTS-plus (table 1).

For analyses of the optimal implementation of DOTS and DOTS-plus we used the baseline probabilities for survival and cure rates (table 1). The probabilities under optimal DOTS-plus differed from those under DOTS in that rates of survival and cure of patients with multidrug resistant tuberculosis were higher under DOTS-plus. In addition, patients treated under DOTS-plus receive second line agents and could therefore develop resistance to these drugs (and consequently develop highly drug resistant tuberculosis), but we assumed that patients treated under DOTS could not..

When we assessed DOTS-plus with decreased levels of effectiveness of the programme, survival and cure rates of multidrug resistant, highly drug resistant, and non-multidrug resistant tuberculosis fell by a percentage of the baseline rate (for example, $5 \%$ ), and the rate of patients developing highly drug resistant tuberculosis rose

\section{Conditions of the analysis}

We accounted for mortality from all causes during the year when tuberculosis was diagnosed, although the rate we used included more than one year of follow up. Death rates for both untreated and treated tuberculosis were for HIV negative patients and were not adjusted for patients' age. We accounted for exogenous reinfection with $M$ tuberculosis by putting patients cured of active disease back in the pool of people at risk for incident tuberculosis. A case of incident tuberculosis became a case of prevalent disease if it had not been cured after one cycle (one year).

\section{Cost effectiveness}

We determined the incremental cost effectiveness of DOTS-plus compared with DOTS by multiplying the marginal added cost of DOTS-plus (compared with DOTS) by the number of patients needed to treat under DOTS-plus to avert one death (compared with treating all patients under DOTS). We determined the number needed to treat by taking the reciprocal of the difference in cumulative deaths under DOTS-plus compared with DOTS. We included only costs related to the patient (such as medicine, medical personnel, and laboratory costs), not costs related to the programme (fixed costs), when determining the marginal added cost. In such a model, local costs can be used to assess local cost effectiveness of DOTS-plus; this is preferred given the variability in costs among programmes to control tuberculosis. In the example used for this analysis, we used marginal cost estimates from India. For DOTS, the marginal cost per patient was $\$ 10(£ 6 ; € 10)$ ( $\mathrm{T}$ R Frieden, unpublished data, 2000). ${ }^{25}$ For DOTS-plus, we assumed that no more than $10 \%$ of patients would receive second line drugs and culture and susceptibility testing. The calculated costs were $\$ 200$ for culture and susceptibility plus $\$ 2000$ for second line agents. ${ }^{26}$ We averaged out the costs of DOTS-plus over all patients: $(0.1 \times \$ 2200)+(0.9 \times \$ 10)=\$ 229$. Thus, the average cost per patient under DOTS-plus would be approximately $\$ 230$ and the marginal added cost of DOTS-plus (compared with DOTS) would be $\$ 230-\$ 10=\$ 220$.

Table 2 Cases of smear positive pulmonary tuberculosis per 100000 population associated with $3 \%$ and $10 \%$ incidence of multidrug resistant tuberculosis

\begin{tabular}{lcc} 
& 3\% incidence & $\begin{array}{c}\mathbf{1 0 \%} \\
\text { incidence }\end{array}$ \\
\hline${\text { Proportion (\%) of incident tuberculosis that is multidrug resistant }{ }^{21-23}}^{23}$ & 3 & 10 \\
\hline Annual incidence $^{22}$ & 100 & 100 \\
\hline Prevalence: $^{22}{ }^{24}$ & 250 & 250 \\
\hline Multidrug resistant tuberculosis $^{21-24}$ & 25 & 100 \\
\hline Non-multidrug resistant tuberculosis $^{24}$ & 225 & 150 \\
\hline
\end{tabular}


Table 3 Cumulative number of deaths (due to prevalent cases of tuberculosis at the start of the analysis and to incident cases during the 10 year period under analysis) from tuberculosis per 100000 population during a 10 year period. Decrements in effectiveness of treatment are compared with baseline DOTS. Numbers in parentheses represent the increase or decrease $(-)$ in the cumulative number of deaths compared with DOTS

\begin{tabular}{|c|c|c|c|c|}
\hline \multirow[b]{2}{*}{ Control programme* } & \multicolumn{3}{|c|}{ Resistance level of Mycobacterium tuberculosis } & \multirow[b]{2}{*}{ Total } \\
\hline & Non-multidrug resistant & Multidrug resistant & Highly drug resistant & \\
\hline \multicolumn{5}{|l|}{$3 \%$ incident tuberculosis } \\
\hline DOTS & 252 & 24 & 0 & 276 \\
\hline \multicolumn{5}{|l|}{ DOTS-plus: } \\
\hline Optimal implementation & $256(4)$ & $16(-8)$ & 0 & $272(-4)$ \\
\hline $5 \%$ decrease in effectiveness of DOTS & $292(40)$ & $20(-4)$ & $8(8)$ & $320(+44)$ \\
\hline $10 \%$ decrease in effectiveness of DOTS & $388(136)$ & $28(4)$ & $4(4)$ & $420(+144)$ \\
\hline \multicolumn{5}{|l|}{$10 \%$ incident tuberculosis } \\
\hline DOTS & 212 & 108 & 0 & 320 \\
\hline \multicolumn{5}{|l|}{ DOTS-plus: } \\
\hline Optimal implementation & $216(4)$ & $68(-40)$ & $4(4)$ & $288(-32)$ \\
\hline $5 \%$ decrease in effectiveness of DOTS & $256(44)$ & $100(-8)$ & $16(16)$ & $372(+52)$ \\
\hline $10 \%$ decrease in effectiveness of DOTS & $316(104)$ & $120(12)$ & $12(12)$ & $448(+128)$ \\
\hline
\end{tabular}

*In a sensitivity analysis that assessed the impact of varying each probability estimate in table 1 by $\pm 10 \%$ from the baseline value, only six variables had any impact on the results, and they were the same for both strategies. In decreasing order of impact on results, the variables were survival rate of treated non-multidrug resistant tuberculosis and prevalent non-multidrug resistant tuberculosis, proportion of patients treated, survival rate of untreated tuberculosis, and cure rates of treated non-multidrug resistant tuberculosis and prevalent non-multidrug resistant tuberculosis. The impact was primarily on deaths from non-multidrug resistant tuberculosis, with minimal to no impact on deaths from multidrug resistant and highly drug resistant tuberculosis. No changes occurred in the relative rank order of deaths (non-multidrug resistant, multidrug resistant, and highly drug resistant tuberculosis) in any of the analyses. The sensitivity analysis therefore did not affect the relative benefit of the DOTS and DOTS-plus strategies, or the conclusions of the analysis.

\section{Results}

\section{Optimal implementation of DOTS}

Based on the probabilities in table 1 for a hypothetical cohort treated with DOTS and for a setting in which the proportion of primary multidrug resistant tuberculosis is 3\% (table 2), 276 deaths per 100000 population would occur during a 10 year period among smear positive cases of pulmonary tuberculosis. Of these, 252 would have non-multidrug resistant and 24 multidrug resistant disease (table 3).

\section{Optimal implementation of DOTS-plus}

Based on the probabilities for DOTS-plus (table 1) and for the same hypothetical cohort and proportion of primary multidrug resistant tuberculosis, four $(1.5 \%)$ fewer patients would die than under DOTS. Eight fewer patients would die with multidrug resistant tuberculosis, but four more would die with nonmultidrug resistant tuberculosis (table 3).

\section{Suboptimal implementation of DOTS-plus}

Since DOTS-plus may not be implemented optimally and its effectiveness would therefore be diminished, we performed the analysis for scenarios in which the survival and cure rates of patients with non-multidrug resistant and multidrug resistant tuberculosis were each $5 \%$ or $10 \%$ less effective than in the DOTS analysis. In addition, the risk of developing highly drug resistant tuberculosis increased with decreasing effectiveness of the programme. If attention to DOTS-plus were to divert resources from DOTS and result in tuberculosis survival and cure rates just 5\% less than those under DOTS, 44 more patients would die with tuberculosis than under DOTS, which represents a $16 \%$ increase in the number of deaths. If DOTS-plus were $10 \%$ less effective than optimal DOTS, 144 additional patients would die compared with DOTS, which represents a $52 \%$ increase (table 3 ).

\section{DOTS and DOTS-plus in "hotspots" of multidrug} resistant tuberculosis

We then compared the effectiveness of DOTS and DOTS-plus in an area where a high proportion (10\%) of cases of incident tuberculosis had multidrug resistance and also adjusted the prevalence of multidrug resistant and non-multidrug resistant tuberculosis (table 2). Under optimal conditions, 40 fewer patients would die with multidrug resistant tuberculosis under DOTS-plus than under DOTS, but also in four deaths due to highly drug resistant tuberculosis that would not have occurred under DOTS. Overall, optimal DOTS-plus would result in $10 \%$ fewer deaths than DOTS. If DOTS-plus were to divert resources from DOTS such that DOTS was just 5\% less effective than under optimal conditions, however, 52 more patients would die from tuberculosis than under baseline DOTS, representing a 16\% increase in the number of deaths (see table 3 ). If the effectiveness of the control programme decreased by $10 \%, 128$ more patients would die with tuberculosis than under DOTS, representing a $40 \%$ increase.

\section{Incremental cost effectiveness of DOTS-plus}

In a setting in which the proportion of primary multidrug resistant tuberculosis is $3 \%$, the number needed to treat under DOTS-plus to avert one death compared with treating all patients under DOTS would be $1 /(276-272) / 1250=313$ patients, where the denominator of 1250 represents prevalent and incident cases per 100000 population with initial treatment over 10 years. Assuming a marginal added cost of DOTS-plus of $\$ 220$, the incremental cost effectiveness ratio would be $\$ 220 \times 313=\$ 68860$ spent for each death averted. In a setting where the proportion of primary multidrug resistant tuberculosis is $10 \%$, the number needed to treat under DOTS-plus would be $1 /(320-288) / 1250=39$ patients, with an incremental cost effectiveness ratio of $\$ 220 \times 39=\$ 8580$.

\section{Discussion}

In an area with $3 \%$ primary multidrug resistant tuberculosis and under optimal implementation of the programme for 10 years, DOTS-plus would result in 1.5\% fewer total deaths from tuberculosis than DOTS, owing to a decrease in the number of deaths due to multidrug 
resistant tuberculosis. Optimal DOTS-plus would therefore lower mortality due to tuberculosis slightly, even in settings with low rates of multidrug resistant tuberculosis. In an area with 10\% primary multidrug resistant tuberculosis and under ideal conditions, DOTS-plus would have a greater impact on lowering total mortality due to tuberculosis ( $10 \%$ fewer deaths) and multidrug resistant tuberculosis. This would be expected since DOTS-plus should have a greater impact in areas with higher rates of multidrug resistant tuberculosis.

The finding is striking, however, that if treatment of tuberculosis in the context of DOTS-plus is only $5 \%$ less effective than optimal DOTS the cumulative number of tuberculosis deaths would be substantially higher under DOTS-plus than under DOTS. DOTSplus would therefore be beneficial as a programme only if it were truly "plus"-if it did not divert resources from DOTS.

\section{Limitations of the study}

This study has several limitations. Firstly, we did not include morbidity due to adverse reactions to the drugs in the analysis. Given the greater toxicity of the second line antituberculosis agents used for DOTS-plus, this would be yet another caution against widespread use of the strategy. Secondly, although the model incorporated the risk of reinfection with $M$ tuberculosis, it did not measure the impact of secondary transmission from people with active tuberculosis. This would tend to underestimate both the potential benefits of DOTS and the potential negative impact of poor implementation. Thirdly, we assumed that highly drug resistant tuberculosis could not develop in settings where second line drugs were not used in the treatment regimen. This may not be true in areas where agents such as fluoroquinolones are widely available. However, the risk of developing highly drug resistant tuberculosis in such settings has not been measured and was therefore not included in the analysis. HIV was not accounted for in the analysis because of insufficient data on the effectiveness of DOTS and DOTS-plus among HIV positive patients. Although HIV infection is associated with an increased risk of tuberculosis among patients infected with $M$ tuberculosis, it is not associated with an increased rate of drug resistant tuberculosis. ${ }^{27}$

\section{Strengths of the study}

Although our baseline analysis assumed that DOTSplus can be implemented effectively, the proportion of patients completing even standard treatment regimens is low in areas where multidrug resistant tuberculosis has become a major problem. ${ }^{21}$ In areas where direct smear microscopy and giving two to four relatively non-toxic drugs for six months is impossible, routinely performing mycobacterial cultures and first and second line susceptibility testing as well as administering four to seven toxic drugs for 18-24 months is unlikely to be possible.

A tuberculosis control programme should have implemented effective DOTS before implementing DOTS-plus. ${ }^{28}$ A poorly run control programme can generate multidrug resistant tuberculosis, but effective DOTS can decrease the rates of multidrug resistant tuberculosis. ${ }^{29}$ More widespread implementation of effective DOTS would therefore decrease the number of cases for which DOTS-plus would be necessary. ${ }^{30}$

\section{What is already known on this topic}

DOTS is an effective, albeit underused, strategy for treating tuberculosis

DOTS may be insufficiently effective in treating multidrug resistant tuberculosis

The use of toxic reserve drugs (DOTS-plus) is an effective but costly strategy for treating multidrug resistant tuberculosis

The impact of the implementation of DOTS-plus on overall tuberculosis control is unknown

\section{What this study adds}

If implementation of DOTS-plus is associated with even minimal decreases in the effectiveness of DOTS, more patients would die with tuberculosis under DOTS-plus than under DOTS alone

If DOTS-plus is implemented, it must not divert resources from and decrease the effectiveness of DOTS

Currently, $77 \%$ of tuberculosis cases worldwide are not treated even with DOTS. ${ }^{8}$

Varying the values of the baseline probability estimates (the sensitivity analysis) did not affect the relative benefit of the DOTS and DOTS-plus strategies or the conclusions of the analysis. This indicates that the findings were relatively robust. The sensitivity analysis also showed that the variables that had the greatest influence on the results were the survival rates of patients with non-multidrug resistant tuberculosis and the proportion of patients who received treatment. This underscores the importance of focusing the programmes' efforts on treating patients with nonmultidrug resistant tuberculosis and ensuring that treatment is successful.

In this analysis the outcome used to assess effectiveness of the programmes was death with tuberculosis because death is a clearly defined outcome that takes into account both the development of active tuberculosis and the effectiveness of treatment. The number of cases of incident tuberculosis is not as instructive because it is not as direct a measure of the effectiveness of treatment (and therefore the effectiveness of tuberculosis control programmes). The model tested in this analysis assumed that the incidence of tuberculosis was the same under all scenarios tested. Although the incidence of tuberculosis could increase in settings where the programme's effectiveness is decreased, this is not supported by available data and was not included in the analysis.

The incremental cost effectiveness ratio in our baseline model for DOTS-plus (\$68 860 to avert one death under DOTS-plus compared with DOTS) is within range of other treatments. However, when the implementation of DOTS-plus leads to reduced effectiveness of DOTS, the DOTS-plus strategy is both less effective and more costly. Given the variation in costs per patient, fixed programme costs, and drug resistance among different geographical regions, as well as population size, further modelling would be 
necessary to make a recommendation for a local jurisdiction.

This analysis does not indicate that DOTS-plus should not be implemented. Rather, it shows the very notable risks associated with implementation of DOTS-plus and shows that, where the strategy is implemented, second line drugs must be used effectively and first line treatment strengthened and insulated from the demands of providing second line drugs on a programme basis.

We thank John Wong for advice related to decision tree design and Christopher Dye, Charles Wells, Thomas Navin, Michael Iademarco, and Zachary Taylor for their review of the manuscript. This paper was presented in part at the American Thoracic Society International Conference in San Francisco, May 2001

Contributors: TRF had the original idea for the study. TRS and HPL designed the decision tree; TRS and TRF researched and decided on the probability estimates. TRS performed the analyses; TRF and HPL critically reviewed the results and provided scientific guidance. TRS wrote the manuscript; TRF and HPL critically reviewed the paper and suggested revisions. All authors approved the final version of the paper. TRS is the guarantor.

Funding: TRS was funded by the National Institute on Allergy and Infectious Diseases (AI01654).

Competing interests: TRF was on loan from the Centers for Disease Control and Prevention to the World Health Organization (Regional Office for South East Asia, New Delhi, India) from 1996-2002 but is no longer affiliated to WHO. The results of this study could benefit WHO because they support WHO recommendations for treating tuberculosis, which could increase funding for the organisation.

1 World Health Organization. Treatment of tuberculosis. Guidelines for national programmes. WHO report. Geneva: WHO, 1997. (WHO/CDS/TB/97.220.)

2 Mitchison DA, Nunn AJ. Influence of initial drug resistance on the response to short-course chemotherapy of pulmonary tuberculosis. $A m$ Rev Respir Dis 1986;133:423-30.

3 Espinal MA, Kim SJ, Suarez PG, Kam KM, Khomenko AG, Migliori GB, et al. Standard short-course chemotherapy for drug-resistant tuberculosis. Treatment outcomes in 6 countries. JAMA 2000;283:2537-45.

4 Farmer P, Kim JY. Community based approaches to the control of multidrug resistant tuberculosis: introducing "DOTS-plus." BMJ 1998;317:671-4.

5 Telzak EE, Sepkowitz K, Alpert P, Mannheimer S, Medard F, El-Sadr W, et al. Multidrug-resistant tuberculosis in patients without HIV infection. $N$ Engl J Med 1995;333:907-11

6 Tahaoglu K, Torun T, Sevim T, Atac G, Kir A, Karasulu L, et al. The treatment of multidrug-resistant tuberculosis in Turkey. $N$ Engl I Med 2001;345:170-4

7 Espinal MA, Dye C, Raviglione M, Kochi A. Rational “DOTS plus” for the control of MDR-TB. Int J Tuberc Lung Dis 1999;3:561-3.
8 World Health Organization. Global tuberculosis control: WHO report. Geneva: WHO, 2001. (WHO/CDS/TB/2001.287.)

9 Beck JR, Pauker SG. The Markov process in medical prognosis. Med Decis Making 1983;3:419-58.

10 Goble M, Iseman MD, Madsen LA, Waite D, Ackerson L, Horsburgh CR Jr. Treatment of 171 patients with pulmonary tuberculosis resistant to isoniazid and rifampicin. N Engl J Med 1993;328:527-32.

11 Kim HJ, Hong YP, Kim SJ, Lew WJ, Lee EG. Ambulatory treatment of multidrug-resistant pulmonary tuberculosis patients at a chest clinic. Int $J$ Tuberc Lung Dis 2001;5:1129-36.

12 Suarez PG, Floyd K, Portocarrero J, Alarcon E, Rapiti E, Ramos G, et al. Feasibility and cost-effectiveness of standardized second-line drug treatment for chronic tuberculosis patients: a national cohort study in Peru. Lancet 2002;359:1980-9.

13 Sullivan EA, Kreiswirth BN, Palumbo L, Kapur V, Musser JM, Ebrahimzadeh A, et al. Emergence of fluoroquinolone-resistant tuberculosis in New York City. Lancet 1995;345:1148-50.

14 Drolet GJ. Present trend of case fatality rates in tuberculosis. Am Rev Tuberc 1938;37:125-51.

15 Thompson BC. Survival rates in pulmonary tuberculosis. BMJ 1943;ii:721.

16 Styblo K. Recent advances in epidemiological research in tuberculosis. Adv Tuberc Res 1980;20:1-63.

17 World Health Organization. Global tuberculosis control: WHO report. Geneva: WHO, 2000. (WHO/CDS/TB/2000.175.)

18 Khatri GR, Frieden TR. The status and prospects of tuberculosis control in India. Int J Tuberc Lung Dis 2000;4:193-200.

19 China Tuberculosis Control Collaboration. Results of directly observed short-course chemotherapy in 112,842 Chinese patients with smearpositive tuberculosis. Lancet 1996;347:358-62.

20 Tuberculosis Research Center, Indian Council of Medical Research. Low rate of emergence of drug resistance in sputum positive patients treated with short-course chemotherapy. Int J Tuberc Lung Dis 2001;5:40-5.

21 Pablos-Mendez A, Raviglione MC, Laszlo A, Binkin N, Rieder HL, Bustreo F, et al. Global surveillance for antituberculosis-drug resistance, 1994-1997. N Engl J Med 1998;338:1641-9.

22 Dye C, Scheele S, Dolin P, Pathania V, Raviglione MC. Global burden of tuberculosis. Estimated incidence, prevalence, and mortality by country. JAMA 1999;282:677-86.

23 Espinal MA, Laszlo A, Simonsen L, Boulahbal F, Kim SJ, Reniero A, et al. Global trends in resistance to antituberculosis drugs. $N$ Engl J Med 2001:344:1294-303.

24 World Health Organization. Global tuberculosis control. WHO Report. Geneva: WHO, 1999. (WHO/TB/99.259.)

25 World Health Organization. Joint tuberculosis programme review, India Geneva: WHO: February 2000. (WHO Project No ICP TUBO.)

26 Gupta R, Kim JY, Espinal MA, Caudron JM, Pecoul B, Farmer PE, Ravigione MC Public health. Responding to market failures in tuberculosis control. Science 2001:293:1049-51.

27 Spellman CW, Matty KJ, Weis SE. A survey of drug-resistant Mycobacterium tuberculosis and its relationship to HIV infection. AIDS 1998:12:191-5.

28 Lambregts-van Weezenbeek KSB, Reichman LB. DOTS and DOTS-Plus: what's in a name. Int J Tuberc Lung Dis 2000;4:995-6.

29 Ledru S, Cauchoix B, Yameogo M, Zoubga A, Lamande-Chiron J, Portaels F, et al. Impact of short-course therapy on tuberculosis drug resistance in south-west Burkina Faso. Tuberc Lung Dis 1996:77:499-36.

30 Dye C, Williams BG, Espinal MA, Raviglione MC. Erasing the world's slow DyeC, Willo BG, Es bea stain: strategies to beat multidrug-resistant tuberculosis. Science

(Accepted 16 December 2002) 